\title{
Correction to: Pheochromocytoma diagnosed during pregnancy: lessons learned from a series of ten patients
}

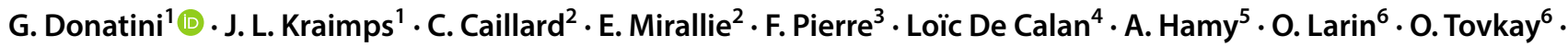 \\ S. Cherenko ${ }^{6}$
}

Published online: 20 March 2018

○) Springer Science+Business Media, LLC, part of Springer Nature 2018

\section{Correction to: Surgical Endoscopy}

https://doi.org/10.1007/s00464-018-6128-x

The original article was updated to correct the listing of A.

Hamy's name; it is correct as displayed above.

The original article can be found online at https://doi.org/10.1007/ s00464-018-6128-x.

G. Donatini

gianluca.donatini@chu-poitiers.fr

1 Department of General and Endocrine Surgery, CHU

Poitiers, 2 Rue de la Miletrie, 86021 Poitiers, France

2 Department of General and Endocrine Surgery, CHU Nantes, Nantes, France

3 Department of Obstetrics and Gynaecology, CHU Poitiers, Poitiers, France

4 Department of General and Endocrine Surgery, CHU Tours, Tours, France

5 Department of General and Endocrine Surgery, CHU Angers, Angers, France

6 Department of Endocrine Surgery, Ukrainian Scientific and Practical Center for Endocrine Surgery of Public Health Ministry of Ukraine, Kiev, Ukraine 